DESY 06-021

WUE-ITP-2006-004

$\mathrm{SFB} / \mathrm{CPP}-06-17$

April 2006

\title{
The planar four-point master integrals for massive two-loop Bhabha scattering
}

\author{
M. Czakon \\ Institut für Theoretische Physik und Astrophysik, Universität Würzburg, \\ Am Hubland, D-97074 Würzburg, Germany, \\ Department of Field Theory and Particle Physics, Institute of Physics, \\ University of Silesia, Uniwersytecka 4, PL-40-007 Katowice, Poland \\ J. Gluza \\ Department of Field Theory and Particle Physics, Institute of Physics, \\ University of Silesia, Uniwersytecka 4, PL-40-007 Katowice, Poland \\ T. Riemann \\ Deutsches Elektronen-Synchrotron, DESY, \\ Platanenallee 6, 15738 Zeuthen, Germany
}

\begin{abstract}
We present the values of the complete set of planar four-point Master Integrals needed for massive Bhabha scattering in the limit of fixed angle and high energy at the two-loop level. The integrals have been calculated using direct expansions of Mellin-Barnes representations, followed by a resummation of resulting harmonic series.
\end{abstract}

\section{Introduction}

The Bhabha scattering process, i.e. the elastic scattering of an electron and a positron is an invaluable tool in the luminosity determination in various experiments. Devices like the International Linear Collider require a theoretical prediction for the cross section that contains, besides 
exponentiated factors coming from the leading logarithmic terms, also fixed order contributions, and in particular the two-loop QED corrections.

As far as large angle Bhabha scattering is concerned a prediction to leading order in the small electron mass (we shall later refer generally to any fermion species) has been derived in [1], although the logarithmic terms have been known before [2]. This prediction has been obtained from the massless result [3] by a careful matching procedure. Since heavy use has been made of results from different sources, it is definitely desirable to obtain the cross section by an independent method. Even though much progress has been made on the way to a result with an exact mass dependence [4,6,5], we are still far from the goal. In the mean time, we have found it much easier to evaluate the required diagrams in the same approximation as in [1]. The present paper presents our results for the set of planar Master Integrals (MI). The main tool are expansions of Mellin-Barnes (MB) integrals.

In the next section we present some considerations on the choice of the MIs, we then describe the derivation and expansion of MB integrals. We subsequently give our results and finish with conclusions. An appendix contains our new MB representations for the five-line integrals.

\section{Master Integrals}

As explained in the introduction, it is our purpose to calculate the integrals in the limit of small fermion mass, which corresponds to high-energy scattering at fixed angle. Since this is a Sudakov approximation, we have to expect an overlap of collinear and on-shell infrared singularities leading to logarithms of the fermion mass reaching power four at the two-loop level. Even though the final result for the cross section will only be given at leading order, i.e. neglecting terms suppressed by $m^{2}$, it is not immediately clear how many terms of the expansion of the individual Master Integrals are needed. Indeed, it is possible that some MIs appear with such coefficients that terms of relative order $1 / \mathrm{m}^{2}$ would result. These would, of course, have to cancel at the end but they would also make a higher order expansion in $m^{2}$ necessary. One way to be sure that the choice of masters avoids this situation is simply to calculate the amplitude and find a set of MIs, whose coefficients would all be of relative order $O(1)$ in the mass expansion. An alternative strategy, which can be applied without knowing the full amplitude is to require that the integrals have their mass dimension given by the kinematic invariants alone. To be specific, we shall require that for any integral $I$, the following relation holds:

$$
I=\left(m^{2}\right)^{-2 \epsilon} s^{n} F\left(\ln \left(-\frac{m^{2}}{s}\right), \frac{t}{s}\right)+O\left(m^{2}\right), \quad n=\frac{1}{2} \operatorname{dim} I+2 \epsilon,
$$

where $s$ and $t$ are the usual Mandelstam kinematic invariants, $\epsilon$ is defined through the dimension of space-time in dimensional regularization, $d=4-2 \epsilon$, and $F$ is some function that we wish to determine. The factor $\left(m^{2}\right)^{-2 \epsilon}$ could have been replaced by $(-s)^{-2 \epsilon}$ through a reshuffling of 
logarithms, but we shall keep it as it is, because at intermediate stages of our calculation the mass has been set to unity for simplification.

Whether Eq. (1) can be satisfied for a given integral or not depends entirely on the strength of the IR singularities. Indeed, usual Feynman graphs have only logarithmic branching points, but putting a dot on some line may result in stronger divergences. This is easily seen in a graph that would have just one massive line regulating the collinear divergence. Putting a dot on it, would be equivalent to a derivative, which transforms a logarithm $\ln ^{n}\left(-m^{2} / s\right)$ into $n \ln ^{n-1}\left(-m^{2} / s\right) / m^{2}$, which is precisely what we want to avoid. Of course, choosing MIs with property Eq. (1) is not yet a proof that there will be no coefficients with $1 / \mathrm{m}^{2}$, but we will be satisfied with the assumption, based on experience, that this is indeed the case.

The consequence of the above considerations is that we have to slightly modify the set of integrals with respect to the original one presented in [5]. In particular, most of the dotted masters have to be replaced by those with irreducible numerators. The new, equivalent set, is given in Section 4. We have, of course, checked that the listed integrals are indeed algebraically independent. In case the reader would like to move back to the old set, we provide the transition formulae in [7].

Having chosen the set of integrals to calculate, there is another question to be considered, namely whether one needs to derive the expansions of the integrals both in the $s$ and in the $t$ channel, as they both occur in the Bhabha scattering amplitude. Fortunately, the limit under study is $s, t \gg m^{2}$, which means that the results are valid in both cases. For consistency of notation, one just has to change

$$
\ln \left(-\frac{m^{2}}{s}\right) \longrightarrow \ln \left(-\frac{m^{2}}{t}\right)=\ln \left(-\frac{m^{2}}{s}\right)-\ln \left(\frac{t}{s}\right)
$$

and

$$
\frac{t}{s} \longrightarrow \frac{s}{t}=1 /\left(\frac{t}{s}\right)
$$

The above transformations imply some algebra in transforming the arguments of the polylogarithms to a unique form. In order to spare the reader the effort, we provide the expansions in both channels in [7].

\section{Expansion of Mellin-Barnes representations}

Mellin-Barnes representations for massive box integrals with the highest number of lines (seven) at the two-loop level have been derived in [8,9]. Since general powers of the propagators have been kept, it should, in principle, be possible to obtain representations for any box integral with a smaller number of lines. The occurrence of factors $1 / \Gamma(a)$, where $a$ is a propagator power, 
seems to make some of the MB integrals trivially vanish. This is actually not the case, since they turn out to be singular in $a$ and an analytic continuation in the relevant propagator power leads to a non-zero result with a smaller dimension of the integral. This procedure is not always optimal and it turns out to be better to derive MB integrals of smaller dimensionality directly for the considered graph. This is particularly true for the 5-line integrals.

There is another case, where direct derivation of representations is necessary. We have seen in the previous section that we need master integrals with irreducible numerators. In such cases it is impossible to obtain the MB integrals directly from the results for the 7-liners. In any case, we derived our own representations by the standard technique of loop-wise integration, with Feynman parameter representations for tensors as given for example in [5]. The new integrals can be found in the Appendix.

Having a MB integral at hand, one has to perform an analytic continuation in $\epsilon$ from a range where the integral is regular to the vicinity of the origin, uncovering the singularity structure on the way. We perform this operation with the MATHEMATICA package MB [10]. As a result, we obtain multidimensional integrals, which still have a nontrivial dependence on both Mandelstam variables. At this stage, the integral can be cast into the following form

$$
I=\left(m^{2}\right)^{-2 \epsilon} \int_{-i \infty}^{i \infty} d z\left(-\frac{m^{2}}{s}\right)^{z} f\left(\frac{t}{s}, z\right)
$$

where the $f$ function contains, amongst others, a product of $\Gamma$, or possibly $\psi$ functions, which have poles in $z$. Let us stress once more that, bar trivial cases, the $f$ function is given by a multidimensional integral. Since it is difficult to directly take residues in this form, we change the order of integration and close the $z$ contour to the right. This procedure is subsequently applied recursively, until no further poles at the required order of expansion occur. Since the MB package can perform numerical integrations, we could check some of the integrals beyond the leading order against the sector decomposition method with relatively large fermion masses. Moreover, we could confirm explicitly that the expansion is given by powers of $m^{2}$ and not of $m$, even though odd powers of the latter are found in individual terms of the series.

Interestingly, the nontrivial dependence on the $t / s$ ratio occurred at the end only in onedimensional integrals. This is in strong contrast to the massless calculation in [11], for example. The one-dimensional integrals were all of the harmonic type and could be done with XSUMMER [12]. The remaining, constant, integrals contained $\Gamma$ functions of doubled argument, $\Gamma(a \pm 2 z)$, which is a trace of the original mass. These have been dealt with high precision numerical integration followed by the use of the PSLQ algorithm [13]. The transcendental constants that could occur in the final result had to be Riemann $\zeta$ numbers up to weight four, because of the correspondence between the expanded massive and the purely massless result. 


\section{Results}

All our integrals are defined with the integration measure

$$
\left(\frac{e^{\epsilon \gamma_{E}}}{i \pi^{2-\epsilon}}\right)^{2} \iint d^{d} k_{1} d^{d} k_{2}
$$

In the presentation of the results, we will use the following notation

$$
x=\frac{t}{s}, \quad L=\ln \left(-\frac{m^{2}}{s}\right)
$$

which guarantees that our results are explicitly real in the Euclidean domain, $s, t<0$; and

$$
\mathrm{S}_{n, p}(z)=\frac{(-1)^{n+p-1}}{(n-1) ! p !} \int_{0}^{1} \frac{d t}{t} \ln ^{n-1}(t) \ln ^{p}(1-z t), \quad \operatorname{Li}_{n}(z)=\mathrm{S}_{n-1,1}(z),
$$

where $S_{n, p}(z)$ is the Nielsen polylogarithm. We will, furthermore, always neglect the $\left(m^{2}\right)^{-2 \epsilon}$ factor in front of the integrals, and keep the $u$ Mandelstam variable in the prefactors, with the understanding that

$$
s+t+u=0
$$

which is correct in the limit under consideration. In fact, we believe that the difficulties encountered in the exact evaluation of the 5-line masters in our previous works are precisely connected

to the occurrence of the $u$ variable in the prefactors, which breaks the simplifications brought by the use of the conformally mapped variables

$$
X=\frac{\sqrt{1-4 m^{2} / s}-1}{\sqrt{1-4 m^{2} / s}+1}, \quad Y=\frac{\sqrt{1-4 m^{2} / t}-1}{\sqrt{1-4 m^{2} / t}+1}
$$

\subsection{7-line integrals}

The two 7-line topologies are given in Fig. 1. Here and in the following we will give the irreducible numerator in parentheses after the integral name. For the 7- and 6-liners we introduce the shorthand

$$
N=\left(k_{2}+p_{4}\right)^{2}
$$




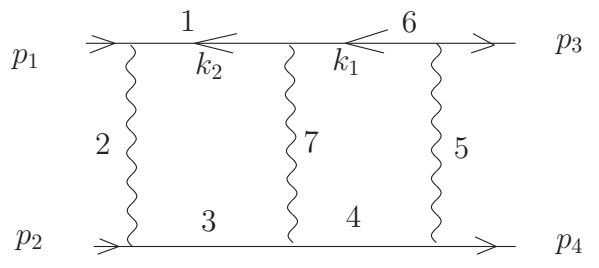

$\mathrm{B} 714 \mathrm{~m} 1$

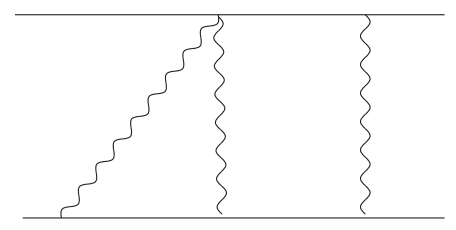

B613m1

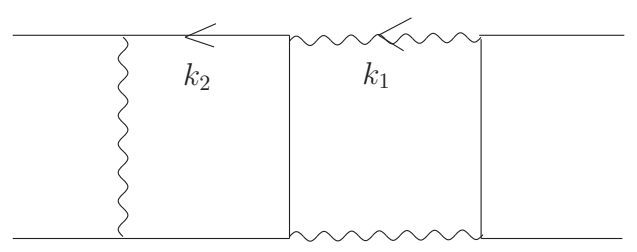

$\mathrm{B} 714 \mathrm{~m} 2$

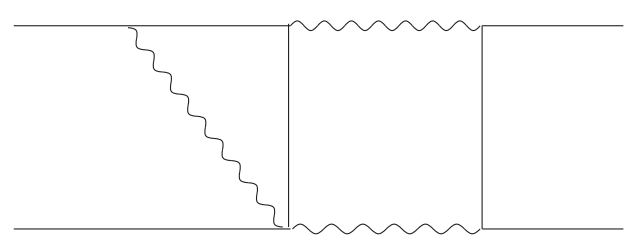

B613m2

Fig. 1. The planar 6- and 7-line topologies. The momentum distribution is common to all integrals.

for the irreducible numerator as in $[8,9]$. For the calculation we used the MB representation from the mentioned papers.

$$
\begin{aligned}
\mathrm{B} 714 \mathrm{~m} 1= & +\frac{1}{\epsilon^{2}} \frac{2 \mathrm{~L}^{2}}{s^{2} t} \\
& -\frac{1}{\epsilon} \frac{1}{3 s^{2} t}\left[-10 \mathrm{~L}^{3}+12 \mathrm{~L}^{2} \ln (x)+6 \mathrm{~L} \zeta_{2}+6 \zeta_{3}\right] \\
& -\frac{1}{6 s^{2} t}\left\{-12 \mathrm{~L}^{4}+28 \mathrm{~L}^{3} \ln (x)-4 \mathrm{~L}^{2}\left(-30 \zeta_{2}+3 \ln ^{2}(x)\right)\right. \\
& -4 \mathrm{~L}\left(9 \zeta_{3}+30 \zeta_{2} \ln (x)+\ln ^{3}(x)-18 \zeta_{2} \ln (1+x)\right. \\
& \left.-3 \ln ^{2}(x) \ln (1+x)-6 \ln (x) \mathrm{Li}_{2}(-x)+6 \mathrm{Li}_{3}(-x)\right) \\
& \left.-15 \zeta_{4}-24 \zeta_{3} \ln (x)\right\} \\
\mathrm{B} 714 \mathrm{~m} 1(\mathrm{~N})= & +\frac{1}{\epsilon^{2}}\left[\frac{3}{2 s^{2}} \mathrm{~L}^{2}\right] \\
& +\frac{1}{\epsilon} \frac{1}{s^{2}}\left[13 / 6 \mathrm{~L}^{3}-2 \mathrm{~L}^{2} \ln (x)-2 \mathrm{~L}_{2}-\zeta_{3}\right] \\
& +\frac{1}{24 s^{2}}\left\{29 \mathrm{~L}^{4}-48 \mathrm{~L}^{3} \ln (x)-408 \mathrm{~L}^{2} \zeta_{2}\right. \\
& +\mathrm{L}\left[168 \zeta_{3}+384 \zeta_{2} \ln (x)+16 \ln ^{3}(x)-288 \zeta_{2} \ln (1+x)\right. \\
& \left.\left.-48 \ln ^{2}(x) \ln (1+x)-96 \ln (x) \mathrm{Li}_{2}(-x)+96 \mathrm{Li}_{3}(-x)\right]+144 \zeta_{4}\right\}
\end{aligned}
$$




$$
\begin{aligned}
\mathrm{B} 714 \mathrm{~m} 2= & -\frac{1}{\epsilon^{2}} \frac{1}{s^{2} t}\left[-\mathrm{L}^{2}+\mathrm{L} \ln (x)\right] \\
& -\frac{1}{\epsilon} \frac{1}{s^{2} t}\left[-2 \mathrm{~L}^{3}+3 \mathrm{~L}^{2} \ln (x)-\mathrm{L} \ln (x)^{2}-\zeta_{2} \ln (x)\right] \\
& -\frac{1}{6 s^{2} t}\left\{-10 \mathrm{~L}^{4}+26 \mathrm{~L}^{3} \ln (x)-2 \mathrm{~L}^{2}\left(-21 \zeta_{2}+9 \ln ^{2}(x)\right)\right. \\
& -2 \mathrm{~L}\left[30 \zeta_{3}+21 \zeta_{2} \ln (x)-\ln ^{3}(x)-18 \zeta_{2} \ln (1+x)\right. \\
& \left.-3 \ln (x)^{2} \ln (1+x)-6 \ln (x) \operatorname{Li}_{2}(-x)+6 \operatorname{Li}_{3}(-x)\right] \\
& \left.+279 \zeta_{4}+18 \zeta_{3} \ln (x)+6 \zeta_{2} \ln ^{2}(x)\right\}
\end{aligned}
$$

$$
\begin{aligned}
\mathrm{B} 714 \mathrm{~m} 2(\mathrm{~N})= & +\frac{1}{\epsilon^{2}} \frac{1}{s^{2}}\left[\mathrm{~L}^{2}-\mathrm{L} \ln (x)\right] \\
& +\frac{1}{\epsilon} \frac{1}{s^{2}}\left[2 \mathrm{~L}^{3}-3 \mathrm{~L}^{2} \ln (x)+\mathrm{L} \ln ^{2}(x)+\zeta_{2} \ln (x)\right] \\
& +\frac{1}{24 s^{2}}\left\{25 \mathrm{~L}^{4}-48 \mathrm{~L}^{3} \ln (x)-456 \mathrm{~L}^{2} \zeta_{2}+\mathrm{L}\left[192 \zeta_{3}+696 \zeta_{2} \ln (x)\right.\right. \\
& +32 \ln ^{3}(x)-288 \zeta_{2} \ln (1+x)-48 \ln ^{2}(x) \ln (1+x) \\
& \left.-96 \ln (x) \operatorname{Li}_{2}(-x)+96 \operatorname{Li}_{3}(-x)\right] \\
& -804 \zeta_{4}-168 \zeta_{3} \ln (x)-216 \zeta_{2} \ln ^{2}(x)-8 \ln ^{4}(x) \\
& +96 \zeta_{2} \ln (x) \ln (1+x)+16 \ln ^{3}(x) \ln (1+x)-192 \zeta_{2} \operatorname{Li}_{2}(-x) \\
& \left.+96 \ln (x) \mathrm{Li}_{3}(-x)-192 \mathrm{Li}_{4}(-x)\right\}
\end{aligned}
$$

$$
\begin{aligned}
\mathrm{B} 714 \mathrm{~m} 2\left(\mathrm{~N}^{2}\right)= & -\frac{1}{\epsilon^{2}} \frac{x}{s}\left[-\mathrm{L}^{2}+\mathrm{L} \ln (x)\right] \\
& -\frac{1}{\epsilon} \frac{1}{s}\left[-2 \mathrm{~L}^{3} x+\mathrm{L}^{2}[3 x \ln (x)-1 / 2]-\mathrm{L} x \ln ^{2}(x)-4 \zeta_{2}-x \zeta_{2} \ln (x)\right] \\
& -\frac{1}{120 s}\left\{-125 \mathrm{~L}^{4} x-5 \mathrm{~L}^{3}[16+56 x-48 x \ln (x)]\right. \\
& -5 \mathrm{~L}^{2}\left[24-456 x \zeta_{2}-144 x \ln (x)\right] \\
& -5 \mathrm{~L}\left[120 \zeta_{2}+384 x \zeta_{2}+192 x \zeta_{3}+696 x \zeta_{2} \ln (x)+96 x \ln ^{2}(x)\right. \\
& +32 x \ln (x)^{3}-288 x \zeta_{2} \ln (1+x)-48 x \ln (x) \ln (1+x) \\
& \left.-96 x \ln (x) \operatorname{Li}_{2}(-x)+96 x \operatorname{Li}_{3}(-x)\right]-960 \zeta_{2}-1920 x \zeta_{2}-840 \zeta_{3} \\
& +480 x \zeta_{3}+4020 x \zeta_{4}+1440 x \zeta_{2} \ln (x)+840 x \zeta_{3} \ln (x) \\
& +1080 x \zeta_{2} \ln { }^{2}(x)+80 x \ln (x)+40 x \ln { }^{4}(x)-1440 x \zeta_{2} \ln (1+x) \\
& -480 x \zeta_{2} \ln (x) \ln (1+x)-240 x \ln ^{2}(x) \ln (1+x) \\
& -80 x \ln (x)^{3} \ln (1+x)+960 x \zeta_{2} \operatorname{Li}_{2}(-x)-480 x \ln (x) \operatorname{Li}_{2}(-x) \\
& \left.+480 x \operatorname{Li}_{3}(-x)-480 x \ln (x) \operatorname{Li}_{3}(-x)+960 x \operatorname{Li}_{4}(-x)\right\}
\end{aligned}
$$




$$
\begin{aligned}
\mathrm{B} 714 \mathrm{~m} 2\left(\mathrm{~N}^{3}\right)= & +\frac{1}{\epsilon^{2}} x^{2}\left[\mathrm{~L}^{2}-\mathrm{L} \ln (x)\right] \\
& +\frac{1}{\epsilon}\left[2 \mathrm{~L}^{3} x^{2}+\mathrm{L}^{2}\left(-1 / 4+x / 2-3 x^{2} \ln (x)\right)+\mathrm{L}\left(1-x+x^{2} \ln ^{2}(x)\right)\right. \\
& \left.-2 \zeta_{2}+4 x \zeta_{2}+x^{2} \zeta_{2} \ln (x)\right] \\
& +\frac{1}{24}\left\{25 \mathrm{~L}^{4} x^{2}+\mathrm{L}^{3}\left(-8+16 x+84 x^{2}-48 x^{2} \ln (x)\right)\right. \\
& +\mathrm{L}^{2}\left(24-72 x^{2}-456 x^{2} \zeta_{2}-216 x^{2} \ln (x)\right) \\
& +\mathrm{L}\left(120-120 x-60 \zeta_{2}+120 x \zeta_{2}+576 x^{2} \zeta_{2}+192 x^{2} \zeta_{3}\right. \\
& +96 x^{2} \ln (x)+696 x^{2} \zeta_{2} \ln (x)+144 x^{2} \ln ^{2}(x)+32 x^{2} \ln ^{3}(x) \\
& -288 x^{2} \zeta_{2} \ln (1+x)-48 x^{2} \ln (x)^{2} \ln (1+x) \\
& \left.\left.-96 x^{2} \ln (x) \mathrm{Li}_{2}(-x)+96 x^{2} \operatorname{Li}_{3}(-x)\right)\right\} \\
& +\left\{\left(3\left(\left(-6+22 x+60 x^{2}\right) \zeta_{2}+(-7+2(7-6 x) x) \zeta_{3}-67 x^{2} \zeta_{4}\right)\right.\right. \\
& +2 x^{2}\left(-\left(\ln (x)\left(54 \zeta_{2}+21 \zeta_{3}+\ln (x)\left(27 \zeta_{2}+\ln (x)(3+\ln (x))\right)\right)\right)\right. \\
& \left.\left.+(9+2 \ln (x))\left(6 \zeta_{2}+\ln ^{2}(x)\right) \ln (1+x)\right)\right) / 6 \\
& -2 x^{2}\left(\left(4 \zeta_{2}-3 \ln (x)\right) \operatorname{Li}_{2}(-x)\right. \\
& \left.\left.+(3-2 \ln (x)) \operatorname{Li}_{3}(-x)+4 \operatorname{Li}_{4}(-x)\right)\right\} \\
& +(-x)
\end{aligned}
$$

\subsection{6-line integrals}

The 6-line integrals are obtained from the 7-line topologies of Fig. 1, by removing line 1 for B613m1 and line 3 for B613m2, and keeping the same momentum distribution. This follows from the fact that we use the same original MB representation from $[8,9]$.

$$
\begin{aligned}
\mathrm{B} 613 \mathrm{~m} 1= & +\frac{1}{\epsilon} \frac{1}{2 s t}\left[\mathrm{~L}^{3}-2 \mathrm{~L}^{2} \ln (x)+\mathrm{L}\left[8 \zeta_{2}+\ln ^{2}(x)\right]\right] \\
& +\frac{1}{24 s t}\left\{7 \mathrm{~L}^{4}-32 \mathrm{~L}^{3} \ln (x)+\mathrm{L}^{2}\left(24 \zeta_{2}+42 \ln ^{2}(x)\right)\right. \\
& +\mathrm{L}\left(24 \zeta_{3}-72 \zeta_{2} \ln (x)-16 \ln ^{3}(x)+72 \zeta_{2} \ln (1+x)\right. \\
& \left.\left.+12 \ln ^{2}(x) \ln (1+x)+24 \ln (x) \operatorname{Li}_{2}(-x)-24 \operatorname{Li}_{3}(-x)\right)\right\} \\
& +\frac{1}{24 s t}\left\{-672 \zeta_{4}-48 \zeta_{2} \ln ^{2}(x)-\ln ^{4}(x)-12\left(6 \zeta_{2}+\ln ^{2}(x)\right) \operatorname{Li}_{2}(-x)\right. \\
& \left.+48 \ln (x) \operatorname{Li}_{3}(-x)-72 \operatorname{Li}_{4}(-x)\right\}
\end{aligned}
$$




$$
\begin{aligned}
\mathrm{B} 613 \mathrm{~m} 1(\mathrm{~N})= & -\frac{1}{\epsilon} \frac{1}{2 s}\left[-\mathrm{L}^{2}-4 \mathrm{~L}+2 \zeta_{2}\right] \\
& -\frac{1}{6 s}\left\{\mathrm{~L}^{3}+\mathrm{L}^{2}(-6-12 \ln (x))\right. \\
& \left.+\mathrm{L}\left(-24+24 \zeta_{2}+6 \ln ^{2}(x)\right)+12 \zeta_{2}+12 \zeta_{3}\right\} \\
\mathrm{B} 613 \mathrm{~m} 2=+ & \frac{1}{24 s t}\left\{7 \mathrm{~L}^{4}-28 \mathrm{~L}^{3} \ln (x)+6 \mathrm{~L}^{2}\left(26 \zeta_{2}+7 \ln ^{2}(x)\right)\right. \\
+ & \mathrm{L}\left(-312 \zeta_{2} \ln ^{2}(x)-28 \ln ^{3}(x)+12\left(6 \zeta_{2}+\ln ^{2}(x)\right) \ln (1+x)\right. \\
+ & \left.24 \ln (x) \mathrm{Li}_{2}(-x)-24 \mathrm{Li}_{3}(-x)\right) \\
+ & 510 \zeta_{4}+156 \zeta_{2} \ln ^{2}(x)+7 \ln ^{4}(x)-8 \ln (x)\left(6 \zeta_{2}+\ln ^{2}(x)\right) \ln (1+x) \\
+ & \left.4\left(6 \zeta_{2}-3 \ln ^{2}(x)\right) \mathrm{Li}_{2}(-x)+24 \mathrm{Li}_{4}(-x)\right\} \\
& \left.+2 \ln (x) \mathrm{Li}_{2}(-x)-2 \mathrm{Li}_{3}(-x)\right\} \\
& -\frac{1}{s}\left\{-9 \zeta_{3}-10 \zeta_{2} \ln ^{2}(x)-\ln ^{3}(x)+6 \zeta_{2} \ln (1+x)+\ln ^{2}(x) \ln (1+x)\right. \\
& -\frac{1}{6 s}\left\{3 \mathrm{~L}^{3}+3 \mathrm{~L}^{2}\left(-2-6 \ln ^{2}(x)\right)+3 \mathrm{~L}\left(18 \zeta_{2}+6 \ln ^{2}(x)\right)\right\} \\
& -\frac{1}{\epsilon} \frac{1}{2 s}\left[-\mathrm{L}^{2}-8 \zeta_{2}\right] \\
& +\mathrm{N})+x
\end{aligned}
$$

\subsection{5-line integrals}

The topologies for the 5-liners with numerators are given in Fig. 2. For the evaluation of the integrals we used our own MB representations given in the Appendix. The position of the dots on the dotted integrals (having a "d" in their name) has been defined in [5]. These integrals belong to our original MI set.

$$
\begin{aligned}
\mathrm{B} 512 \mathrm{~m} 1= & +\frac{1}{\epsilon^{2}} \frac{1}{s}[\mathrm{~L}] \\
& -\frac{1}{\epsilon} \frac{1}{s}\left[-3 / 2 \mathrm{~L}^{2}+\mathrm{L}(-2+\ln (x))+\zeta_{2}\right] \\
& -\frac{1}{6 s}\left\{-7 \mathrm{~L}^{3}+\mathrm{L}^{2}(-18+9 \ln (x))+\mathrm{L}\left(-24+24 \zeta_{2}+12 \ln (x)-3 \ln ^{2}(x)\right)\right. \\
& +12 \zeta_{2}+12 \zeta_{3}-24 \zeta_{2} \ln (x)-\ln ^{3}(x)+18 \zeta_{2} \ln (1+x) \\
& \left.+3 \ln ^{2}(x) \ln (1+x)+6 \ln (x) \operatorname{Li}_{2}(-x)-6 \operatorname{Li}_{3}(-x)\right\}
\end{aligned}
$$




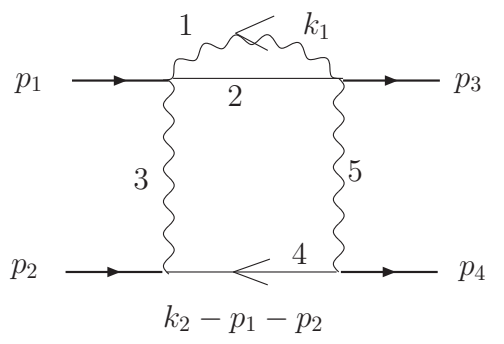

$\mathrm{B} 512 \mathrm{~m} 2$

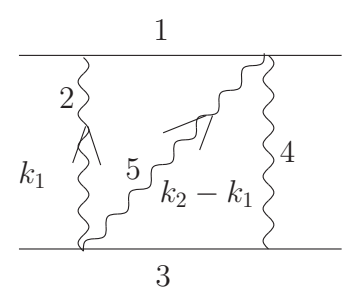

B $512 \mathrm{~m} 3$

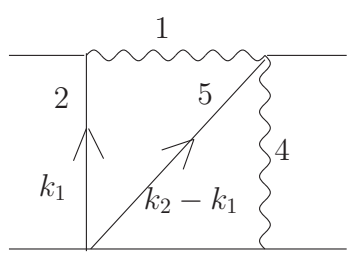

3

Fig. 2. The 5-line topologies. The momentum distribution has been chosen to make the derivation of MB representations easier.

$$
\begin{aligned}
\mathrm{B} 512 \mathrm{~m} 2= & -\frac{1}{\epsilon} \frac{1}{6 t}\left[-3 \mathrm{~L}^{2}+6 \mathrm{~L} \ln (x)-3 \ln ^{2}(x)-24 \zeta_{2}\right] \\
& -\frac{1}{6 t}\left\{-4 \mathrm{~L}^{3}-\mathrm{L}^{2}(6-9 \ln (x))-\mathrm{L}\left(6 \zeta_{2}-12 \ln (x)+6 \ln ^{2}(x)\right)\right. \\
& -48 \zeta_{2}-54 \zeta_{3}-18 \zeta_{2} \ln (x)-6 \ln ^{2}(x)+\ln ^{3}(x) \\
& +18 \zeta_{2} \ln (1+x)+3 \ln ^{2}(x) \ln (1+x) \\
& \left.+6 \ln (x) \operatorname{Li}_{2}(-x)-6 \operatorname{Li}_{3}(-x)\right\}
\end{aligned}
$$

$$
\begin{aligned}
\mathrm{B} 512 \mathrm{~m} 2\left(\mathrm{k}_{2} \cdot \mathrm{p}_{3}\right)= & +\frac{1}{\epsilon} \frac{s}{12 t}\left[3 \mathrm{~L}^{2}+\mathrm{L}(12+6 x-6 \ln (x))\right. \\
+ & \left.24 \zeta_{2}-12 \ln (x)-6 x \ln (x)+3 \ln ^{2}(x)\right] \\
& +\frac{s}{12 t}\left\{4 \mathrm{~L}^{3}+\mathrm{L}^{2}(3(8+3 x)-9 \ln (x))\right. \\
+ & \mathrm{L}\left[6\left(10+5 x+\zeta_{2}\right)-12(3+x) \ln (x)+6 \ln ^{2}(x)\right] \\
+ & 12 \zeta_{2}+54 \zeta_{3}-60 \ln (x)-30 x \ln (x)+18 \zeta_{2} \ln (x)+12 \ln ^{2}(x) \\
& +6 x \ln ^{2}(x)-\ln ^{3}(x)-18 \zeta_{2} \ln (1+x)-3 \ln ^{2}(x) \ln (1+x) \\
& \left.-6 \ln (x) \mathrm{Li}_{2}(-x)+6 \operatorname{Li}_{3}(-x)\right\}
\end{aligned}
$$

$$
\begin{aligned}
\mathrm{B} 512 \mathrm{~m} 2 \mathrm{~d} 2= & +\frac{1}{6 s t}\left\{-12 \mathrm{~L}^{3}+9 \mathrm{~L}^{2} \ln (x)-36 \mathrm{~L} \zeta_{2}\right. \\
& -12 \zeta_{3}-12 \zeta_{2} \ln (x)-\ln ^{3}(x)+18 \zeta_{2} \ln (1+x) \\
& \left.+3 \ln ^{2}(x) \ln (1+x)+6 \ln (x) \operatorname{Li}_{2}(-x)-6 \operatorname{Li}_{3}(-x)\right\}
\end{aligned}
$$

$$
\mathrm{B} 512 \mathrm{~m} 3=+\frac{1}{12 u}\left\{-6 \mathrm{~L}^{2}\left(6 \zeta_{2}+\ln ^{2}(x)\right)\right.
$$




$$
\begin{aligned}
& -6 \mathrm{~L}\left(-4 \zeta_{3}+4 \zeta_{2} \ln (x)-12 \zeta_{2} \ln (1+x)-2 \ln ^{2}(x) \ln (1+x)\right. \\
& \left.-4 \ln (x) \mathrm{Li}_{2}(-x)+4 \mathrm{Li}_{3}(-x)\right) \\
& +312 \zeta_{4}+72 \zeta_{3} \ln (x)+36 \zeta_{2} \ln ^{2}(x)+\ln ^{4}(x)-24 \zeta_{3} \ln (1+x) \\
& +24 \zeta_{2} \ln (x) \ln (1+x)-36 \zeta_{2} \ln ^{2}(1+x)-6 \ln ^{2}(x) \ln ^{2}(1+x) \\
& -24 \ln (x) \mathrm{S}_{1,2}(-x)+12\left(8 \zeta_{2}+\ln ^{2}(x)-2 \ln (x) \ln (1+x)\right) \mathrm{Li}_{2}(-x) \\
& -48 \ln (x) \mathrm{Li}_{3}(-x)+24 \ln (1+x) \mathrm{Li}_{3}(-x) \\
& \left.+72 \mathrm{Li}_{4}(-x)+24 \mathrm{~S}_{2,2}(-x)\right\}
\end{aligned}
$$

$$
\begin{aligned}
\mathrm{B} 512 \mathrm{~m} 3 \mathrm{~d} 2= & -\frac{1}{\epsilon^{2}} \frac{1}{s t} \mathrm{~L} \\
& +\frac{1}{\epsilon} \frac{1}{s t}\left[-3 \mathrm{~L}^{2}+2 \mathrm{~L} \ln (x)-\zeta_{2}\right] \\
& +\frac{1}{3 s t}\left\{-10 \mathrm{~L}^{3}+12 \mathrm{~L}^{2} \ln (x)+51 \mathrm{~L} \zeta_{2}\right. \\
& -21 \zeta_{3}-42 \zeta_{2} \ln (x)-2 \ln ^{3}(x)+36 \zeta_{2} \ln (1+x) \\
& \left.+6 \ln ^{2}(x) \ln (1+x)+12 \ln (x) \operatorname{Li}_{2}(-x)-12 \operatorname{Li}_{3}(-x)\right\} \\
& +\epsilon \frac{1}{3 s t}\left\{-5 \mathrm{~L}^{4}+10 \mathrm{~L}^{3} \ln (x)+111 \mathrm{~L}^{2} \zeta_{2}\right. \\
& +\mathrm{L}\left(104 \zeta_{3}-126 \zeta_{2} \ln (x)-6 \ln ^{3}(x)+108 \zeta_{2} \ln (1+x)\right. \\
& \left.+18 \ln ^{2}(x) \ln (1+x)+36 \ln (x) \operatorname{Li}_{2}(-x)-36 \operatorname{Li}_{3}(-x)\right) \\
& -372 \zeta_{4}-78 \zeta_{3} \ln (x)+30 \zeta_{2} \ln ^{2}(x)+2 \ln ^{4}(x)+12 \zeta_{3} \ln (1+x) \\
& -60 \zeta_{2} \ln (x) \ln (1+x)-8 \ln ^{3}(x) \ln (1+x)+18 \zeta_{2} \ln ^{2}(1+x) \\
& +3 \ln ^{2}(x) \ln ^{2}(1+x)+12 \ln (x) \mathrm{S}_{1,2}(-x) \\
& -6\left(4 \zeta_{2}+3 \ln ^{2}(x)-2 \ln (x) \ln ^{2}(1+x)\right) \operatorname{Li}_{2}(-x) \\
& +24 \ln ^{2}(x) \operatorname{Li}_{3}(-x)-12 \ln (1+x) \operatorname{Li}_{3}(-x) \\
& \left.-12 \operatorname{Li}_{4}(-x)-12 \mathrm{~S}_{2,2}(-x)\right\}
\end{aligned}
$$

$$
\begin{aligned}
\mathrm{B} 512 \mathrm{~m} 3\left(\mathrm{k}_{2} \cdot \mathrm{p}_{2}\right) & =\frac{1}{4}\left(\frac{s}{u}\right)^{2}\left\{\mathrm{~L}^{2}\left(6 x \zeta_{2}+2 x \ln (x)+2 x^{2} \ln (x)+x \ln ^{2}(x)\right)\right. \\
& +\mathrm{L}\left(16 x \zeta_{2}-8 x^{2} \zeta_{2}-4 x \zeta_{3}-2 \ln (x)+2 x^{2} \ln (x)\right. \\
& +4 x \zeta_{2} \ln (x)+2 x \ln ^{2}(x)-2 x^{2} \ln ^{2}(x)-12 x \zeta_{2} \ln (1+x) \\
& \left.\left.-2 x \ln ^{2}(x) \ln (1+x)-4 x \ln (x) \mathrm{Li}_{2}(-x)+4 x \operatorname{Li}_{3}(-x)\right)\right\} \\
& +\frac{1}{120}\left(\frac{s}{u}\right)^{2}\left\{+120 \zeta_{2}+360 x \zeta_{2}-120 x^{2} \zeta_{2}-1560 x \zeta_{4}-480 x \zeta_{3}\right. \\
& -240 x^{2} \zeta_{3}-240 x \zeta_{2} \ln (x)-480 x^{2} \zeta_{2} \ln (x)-360 x \zeta_{3} \ln (x)
\end{aligned}
$$




$$
\begin{aligned}
& +30 \ln ^{2}(x)+60 x \ln ^{2}(x)-30 x^{2} \ln ^{2}(x)-180 x \zeta_{2} \ln ^{2}(x) \\
& -20 x \ln ^{3}(x)-20 x^{2} \ln ^{3}(x)-5 x \ln ^{4}(x)+720 x^{2} \zeta_{2} \ln (1+x) \\
& +120 x \zeta_{3} \ln (1+x)-120 x \zeta_{2} \ln (x) \ln (1+x)+120 x^{2} \ln ^{2}(x) \ln (1+x) \\
& +180 x \zeta_{2} \ln ^{2}(1+x)+30 x \ln ^{2}(x) \ln ^{2}(1+x)+120 x \ln (x) \mathrm{S}_{1,2}(-x) \\
& +60 x\left(-8 \zeta_{2}-\ln ^{2}(x)+2 \ln (x)(2 x+\ln (1+x))\right) \operatorname{Li}_{2}(-x) \\
& -240 x^{2} \operatorname{Li}_{3}(-x)+240 x \ln (x) \operatorname{Li}_{3}(-x)-120 x \ln (1+x) \operatorname{Li}_{3}(-x) \\
& \left.-360 x \operatorname{Li}_{4}(-x)-120 x \mathrm{~S}_{2,2}(-x)\right\}
\end{aligned}
$$

$$
\begin{aligned}
& \mathrm{B} 512 \mathrm{~m} 3\left(\mathrm{k}_{2} \cdot \mathrm{p}_{3}\right)=\frac{1}{4}\left(\frac{s}{u}\right)^{2}\left\{\mathrm { L } ^ { 2 } \left(12 x \zeta_{2}+6 x^{2} \zeta_{2}-2 \ln (x)-2 x \ln (x)+2 x \ln ^{2}(x)\right.\right. \\
& \left.+x^{2} \ln ^{2}(x)\right)+\mathrm{L}\left(-4 \zeta_{2}+20 x \zeta_{2}-8 x \zeta_{3}-4 x^{2} \zeta_{3}\right. \\
& -2 \ln (x)+2 x^{2} \ln (x)+8 x \zeta_{2} \ln (x)+4 x^{2} \zeta_{2} \ln (x) \\
& +4 x \ln ^{2}(x)-24 x \zeta_{2} \ln (1+x)-12 x^{2} \zeta_{2} \ln (1+x) \\
& -4 x \ln ^{2}(x) \ln (1+x)-2 x^{2} \ln ^{2}(x) \ln (1+x) \\
& \left.\left.-4 x(2+x) \ln (x) \operatorname{Li}_{2}(-x)+4 x(2+x) \operatorname{Li}_{3}(-x)\right)\right\} \\
& +\frac{1}{24}\left(\frac{s}{u}\right)^{2}\left\{-24(-1+(-3+x) x) \zeta_{2}+72 \zeta_{3}+24 x \zeta_{3}-552 x(2+x) \zeta_{4}\right. \\
& -48 x(2+x) \zeta_{3} \ln (x)+\ln (x)\left(48(1+x) \zeta_{2}\right. \\
& +\ln (x)\left(6-6 x\left(-2+x+16 \zeta_{2}+8 x \zeta_{2}\right)+4(1+x) \ln (x)\right. \\
& \left.\left.-x(2+x) \ln ^{2}(x)\right)\right)+24 x(2+x) \zeta_{3} \ln (1+x) \\
& +4\left(-3(1+3 x)\left(6 \zeta_{2}+\ln ^{2}(x)\right)+\ln (-x)\left(24 x(2+x) \zeta_{2}\right.\right. \\
& +3 \ln (x)(2+6 x+x(2+x) \ln (x)))) \ln (1+x) \\
& +6 x(2+x)\left(6 \zeta_{2}-2 \ln (-x) \ln (x)+\ln ^{2}(x)\right) \ln ^{2}(1+x) \\
& +96 x(2+x) \zeta_{2}\left(\zeta_{2}-\ln (-x) \ln (1+x)-\operatorname{Li}_{2}(-x)\right) \\
& +24 \ln (x)\left(\zeta_{2}-\ln (-x) \ln (1+x)-\operatorname{Li}_{2}(-x)\right) \\
& +72 x \ln (x)\left(\zeta_{2}-\ln (-x) \ln (1+x)-\operatorname{Li}_{2}(-x)\right) \\
& +12 x(2+x) \ln ^{2}(x)\left(\zeta_{2}-\ln (-x) \ln (1+x)-\operatorname{Li}_{2}(-x)\right) \\
& +24 \mathrm{Li}_{3}(-x)+72 x \mathrm{Li}_{3}(-x)+48 x(2+x) \ln (x) \mathrm{Li}_{3}(-x) \\
& -24 x(2+x) \ln (1+x) \operatorname{Li}_{3}(-x)-12 x(2+x)\left(2 \zeta_{3} \ln (x)\right. \\
& +2 \zeta_{2} \ln (x) \ln (1+x)-\ln (-x) \ln (x) \ln ^{2}(1+x)-2 \ln (x) \mathrm{S}_{1,2}(-x) \\
& \left.\left.-2 \ln (x) \ln (1+x) \operatorname{Li}_{2}(-x)+6 \operatorname{Li}_{4}(-x)+2 \mathrm{~S}_{2,2}(-x)\right)\right\}
\end{aligned}
$$

$$
\begin{aligned}
\mathrm{B} 513 \mathrm{~m}= & +\frac{1}{6 u}\left\{\mathrm{~L}^{2}\left(-18 \zeta_{2}-3 \ln ^{2}(x)\right)+\mathrm{L}\left(12 \zeta_{3}+2 \ln ^{3}(x)+36 \zeta_{2} \ln (1+x)\right.\right. \\
& \left.+6 \ln ^{2}(x) \ln (1+x)+12 \ln (x) \mathrm{Li}_{2}(-x)-12 \mathrm{Li}_{3}(-x)\right)
\end{aligned}
$$




$$
\begin{aligned}
& -81 \zeta_{4}-12 \zeta_{3} \ln (1+x)-2 \ln ^{3}(x) \ln (1+x)-18 \zeta_{2} \ln ^{2}(1+x) \\
& -3 \ln ^{2}(x) \ln ^{2}(1+x)-12 \ln (x) \mathrm{S}_{1,2}(-x)-6 \ln (x)(\ln (x) \\
& +2 \ln (1+x)) \operatorname{Li}_{2}(-x)+12 \ln (x) \operatorname{Li}_{3}(-x)+12 \ln (1+x) \operatorname{Li}_{3}(-x) \\
& \left.-12 \operatorname{Li}_{4}(-x)+12 \mathrm{~S}_{2,2}(-x)\right\} \\
\text { B513md3 }= & +\frac{1}{\epsilon} \frac{1}{s t}\left[-\mathrm{L}^{2}+\mathrm{L} \ln (x)\right] \\
& +\frac{m_{s}^{2}}{3 x}\left\{-8 \mathrm{~L}^{3}+12 \mathrm{~L}^{2} \ln (x)+\mathrm{L}\left(24 \zeta_{2}-3 \ln ^{2}(x)\right)\right. \\
& -21 \zeta_{2} \ln (x)-\ln ^{3}(x)+18 \zeta_{2} \ln (1+x)+3 \ln ^{2}(x) \ln (1+x) \\
& \left.+6 \ln (x) \operatorname{Li}_{2}(-x)-6 \operatorname{Li}_{3}(-x)\right\}
\end{aligned}
$$

$$
\begin{aligned}
\mathrm{B} 513 \mathrm{~m}\left(\mathrm{k}_{1} \cdot \mathrm{p}_{2}\right)= & +\frac{1}{12}\left(\frac{s}{u}\right)^{2}\{ \\
& +\mathrm{L}^{2}\left(-18 x \zeta_{2}+6 \ln (x)+6 x \ln (x)-3 x \ln (x)^{2}\right) \\
& +\mathrm{L}\left(36 \zeta_{2}-36 x \zeta_{2}+12 x \zeta_{3}+6 \ln (x)-6 x^{2} \ln (x)\right. \\
& -12 x \ln (x)^{2}+2 x \ln (x)^{3}+36 x \zeta_{2} \ln (1+x)+6 x \ln (x)^{2} \ln (1+x) \\
& \left.+12 x \ln (x) \mathrm{Li}_{2}(-x)-12 x \mathrm{Li}_{3}(-x)\right)+12 \zeta_{2}-12 x \zeta_{2} \\
& +12 x^{2} \zeta_{2}-12 \zeta_{3}+12 x \zeta_{3}-81 x \zeta_{4}-3 \ln (x)^{2}-6 x \ln (x)^{2} \\
& +3 x^{2} \ln (x)^{2}-2 \ln (x)^{3}+2 x \ln (x)^{3}-36 \zeta_{2} \ln (1+x) \\
& +36 x \zeta_{2} \ln (1+x)-12 x \zeta_{3} \ln (1+x)-6 \ln (x)^{2} \ln (1+x) \\
& +6 x \ln (x)^{2} \ln (1+x)-2 x \ln (x)^{3} \ln (1+x)-18 x \zeta_{2} \ln (1+x)^{2} \\
& -3 x \ln (x)^{2} \ln (1+x)^{2}-12 x \ln (x) \mathrm{S}_{1,2}(-x)-6 \ln (x)(2-2 x+x \ln (x) \\
& +2 x \ln (1+x)) \operatorname{Li}_{2}(-x)+12 \operatorname{Li}_{3}(-x)-12 x \operatorname{Li}_{3}(-x)+12 x \ln (x) \operatorname{Li}_{3}(-x) \\
& \left.+12 x \ln (1+x) \operatorname{Li}_{3}(-x)-12 x \operatorname{Li}_{4}(-x)+12 x \mathrm{~S}_{2,2}(-x)\right\}
\end{aligned}
$$

$$
\begin{aligned}
\mathrm{B} 513 \mathrm{~m}\left(\mathrm{k}_{1} \cdot \mathrm{p}_{3}\right)= & +\frac{1}{12}\left(\frac{s}{u}\right)^{2}\{ \\
& +\mathrm{L}^{2}\left(18 x^{2} \zeta_{2}-6 x \ln (x)-6 x^{2} \ln (x)+3 x^{2} \ln (x)^{2}\right) \\
& +\mathrm{L}\left(-72 x \zeta_{2}-12 x^{2} \zeta_{3}+6 \ln (x)-6 x^{2} \ln (x)-6 x \ln (x)^{2}\right. \\
& +6 x^{2} \ln (x)^{2}-2 x^{2} \ln (x)^{3}-36 x^{2} \zeta_{2} \ln (1+x)-6 x^{2} \ln (x)^{2} \ln (1+x) \\
& \left.-12 x^{2} \ln (x) \mathrm{Li}_{2}(-x)+12 x^{2} \mathrm{Li}_{3}(-x)\right)-12 \zeta_{2}-60 x \zeta_{2}-12 x^{2} \zeta_{2} \\
& +24 x \zeta_{3}+81 x^{2} \zeta_{4}-12 x \zeta_{2} \ln (x)-12 x^{2} \zeta_{2} \ln (x)-3 \ln (x)^{2} \\
& -6 x \ln (x)^{2}+3 x^{2} \ln (x)^{2}+2 x \ln (x)^{3}-2 x^{2} \ln (x)^{3} \\
& +72 x \zeta_{2} \ln (1+x)+12 x^{2} \zeta_{3} \ln (1+x)+12 x \ln (x)^{2} \ln (1+x) \\
& +2 x^{2} \ln (x)^{3} \ln (1+x)+18 x^{2} \zeta_{2} \ln (1+x)^{2}+3 x^{2} \ln (x)^{2} \ln (1+x)^{2}
\end{aligned}
$$




$$
\begin{aligned}
& +12 x^{2} \ln (x) \mathrm{S}_{1,2}(-x)+6 x \ln (x)(4+x \ln (x)+2 x \ln (1+x)) \mathrm{Li}_{2}(-x) \\
& -24 x \mathrm{Li}_{3}(-x)-12 x^{2} \ln (x) \operatorname{Li}_{3}(-x)-12 x^{2} \ln (1+x) \operatorname{Li}_{3}(-x) \\
& \left.+12 x^{2} \operatorname{Li}_{4}(-x)-12 x^{2} \mathrm{~S}_{2,2}(-x)\right\}
\end{aligned}
$$

$$
\begin{aligned}
\mathrm{B} 513 \mathrm{~m}\left(\mathrm{k}_{2} \cdot \mathrm{p}_{2}\right)= & +\frac{1}{12}\left(\frac{s}{u}\right)^{2}\{ \\
& +\mathrm{L}^{2}\left(18 x \zeta_{2}+6 x \ln (x)+6 x^{2} \ln (x)+3 x \ln ^{2}(x)\right) \\
& +\mathrm{L}\left(36 x \zeta_{2}-36 x^{2} \zeta_{2}-12 x \zeta_{3}-6 \ln (x)+6 x^{2} \ln (x)-12 x^{2} \ln ^{2}(x)\right. \\
& -2 x \ln ^{3}(x)-36 x \zeta_{2} \ln (1+x)-6 x \ln ^{2}(x) \ln (1+x) \\
& \left.-12 x \ln (x) \operatorname{Li}_{2}(-x)+12 x \operatorname{Li}_{3}(-x)\right)-12 \zeta_{2}+12 x \zeta_{2} \\
& -12 x^{2} \zeta_{2}-12 x \zeta_{3}+12 x^{2} \zeta_{3}+81 x \zeta_{4}+3 \ln ^{2}(x) \\
& +6 x \ln ^{2}(x)-3 x^{2} \ln ^{2}(x)-2 x \ln ^{3}(x)+2 x^{2} \ln ^{3}(x)-36 x \zeta_{2} \ln (1+x) \\
& +36 x^{2} \zeta_{2} \ln (1+x)+12 x \zeta_{3} \ln (1+x)-6 x \ln ^{2}(x) \ln (1+x) \\
& +6 x^{2} \ln ^{2}(x) \ln (1+x)+2 x \ln 3(x) \ln (1+x)+18 x \zeta_{2} \ln ^{2}(1+x) \\
& +3 x \ln ^{2}(x) \ln { }^{2}(1+x)+12 x \ln (x) \mathrm{S}_{1,2}(-x)+6 x \ln (x)(\ln (x) \\
& +2(-1+x+\ln (1+x))) \operatorname{Li}_{2}(-x)+12 x \operatorname{Li}_{3}(-x)-12 x^{2} \operatorname{Li}_{3}(-x) \\
& -12 x \ln (x) \operatorname{Li}_{3}(-x)-12 x \ln (1+x) \operatorname{Li}_{3}(-x) \\
& \left.+12 x \operatorname{Li}_{4}(-x)-12 x \mathrm{~S}_{2,2}(-x)\right\} \\
& -33)
\end{aligned}
$$

$$
\begin{aligned}
\mathrm{B} 514 \mathrm{~m}= & +\frac{1}{\epsilon^{2}} \frac{1}{s}[\mathrm{~L}] \\
& -\frac{1}{\epsilon} \frac{1}{s}\left[-3 / 2 \mathrm{~L}^{2}+\mathrm{L}(-2+\ln (x))+\zeta_{2}\right] \\
& -\frac{1}{6 s}\left\{-7 \mathrm{~L}^{3}+\mathrm{L}^{2}(-18+9 \ln (x))+\mathrm{L}\left(-24+24 \zeta_{2}+12 \ln (x)-3 \ln ^{2}(x)\right)\right. \\
& +12 \zeta_{2}+12 \zeta_{3}-24 \zeta_{2} \ln (x)-\ln ^{3}(x)+18 \zeta_{2} \ln (1+x) \\
& \left.+3 \ln ^{2}(x) \ln (1+x)+6 \ln (x) \operatorname{Li}_{2}(-x)-6 \operatorname{Li}_{3}(-x)\right\}
\end{aligned}
$$

$$
\begin{aligned}
\text { B514md } & =\frac{1}{6 s t}\left\{-11 \mathrm{~L}^{3}+21 \mathrm{~L}^{2} \ln (x)+\mathrm{L}\left(30 \zeta_{2}-9 \ln ^{2}(x)\right)\right. \\
& -24 \zeta_{2} \ln (x)-\ln ^{3}(x)+18 \zeta_{2} \ln (1+x)+3 \ln ^{2}(x) \ln (1+x) \\
& \left.+6 \ln (x) \mathrm{Li}_{2}(-x)-6 \operatorname{Li}_{3}(-x)\right\}
\end{aligned}
$$




\section{Conclusions}

With the help of a direct expansion of MB integrals, we obtained all of the planar box masters to leading order in the expansion in the small fermion mass. The calculation has been substantially simplified by the fact that all nontrivial integrations were one-dimensional. Unfortunately, the remaining, non-planar MIs introduce two further complications. First, the final integrals are not directly one-dimensional. This is per se not an insurmountable obstacle, since a similar situation has been dealt with during the evaluation of the fully massless masters. However, in our case it seems non-trivial to bring the integrals to the form of harmonic sums. The second problem is connected to the fact, that MB representations for the non-planar case involve all three kinematic invariants. All in all, despite the above problems we hope that the technique used in this paper will prove successful also in this case, and we will be able to provide the complete Bhabha scattering cross section in the limit of high energy and fixed angle from a direct diagrammatic calculation and thus verify results obtained by matching between the massive and massless cases [1]. Moreover, our approach can be applied to problems with more scales, in particular, we have applied it to the integrals occuring in the Bhabha cross section with two fermion species [14].

\section{Acknowledgments}

We would like to thank Sven Moch for useful discussions. Work supported in part by Sonderforschungsbereich/Transregio 9-03 of DFG 'Computergestützte Theoretische Teilchenphysik', by the Sofja Kovalevskaja Award of the Alexander von Humboldt Foundation sponsored by the German Federal Ministry of Education and Research, and by the Polish State Committee for Scientific Research (KBN), research projects in 2004-2005.

\section{A Mellin-Barnes representations for 5-line integrals}

This appendix contains the MB representations that we directly derived for the 5-line integrals with as well without irreducible numerators. Notice that the momentum $p_{e}$ can be any of the external momenta, because we performed the integration with a single loop momentum in the

numerator with vector index kept free. As far as the notation is concerned, the $a_{i}$ denote the power of line $i$. The numbering can be read off Fig. 2.

$$
\begin{aligned}
\mathrm{B} 512 \mathrm{~m} 2= & \frac{(-1)^{a_{12345}} e^{2 \epsilon \gamma_{E}}}{\prod_{j=1}^{5} \Gamma\left[a_{i}\right] \Gamma\left[4-2 \epsilon-a_{13}\right](2 \pi i)^{3}} \int_{-i \infty}^{+i \infty} d \alpha \int_{-i \infty}^{+i \infty} d \beta \int_{-i \infty}^{+i \infty} d \gamma \\
& (-s)^{2-\epsilon-a_{245}-\gamma-\alpha+\beta}(-t)^{\alpha} \Gamma\left[-2+\epsilon+a_{13}+\beta\right] \\
& \Gamma[-\gamma] \Gamma\left[2-\epsilon-a_{245}-\gamma-\alpha\right] \Gamma[-\alpha]
\end{aligned}
$$




$$
\begin{aligned}
& \Gamma\left[a_{2}+\alpha\right] \Gamma\left[a_{4}+\alpha\right] \Gamma\left[4-2 \epsilon-a_{113}-\beta\right] \\
& \Gamma\left[-2+\epsilon+a_{245}+\gamma+\alpha-\beta\right] \Gamma\left[a_{1}+\beta\right] \\
& \frac{\Gamma\left[4-2 \epsilon-a_{2245}-2 \alpha+\beta\right] \Gamma\left[2-\epsilon-a_{24}-\gamma-\alpha+\beta\right]}{\Gamma\left[4-2 \epsilon-a_{245}+\beta\right] \Gamma\left[4-2 \epsilon-a_{22445}-2 \gamma-2 \alpha+\beta\right]}
\end{aligned}
$$

$$
\begin{aligned}
\mathrm{B} 512 \mathrm{~m} 2\left(\mathrm{p}_{\mathrm{e}} \cdot \mathrm{k}_{2}\right)= & \frac{(-1)^{a_{12345}-1} e^{2 \epsilon \gamma_{E}}}{\Gamma\left[a_{1}\right] \Gamma\left[a_{2}\right] \Gamma\left[a_{3}\right] \Gamma\left[a_{4}\right] \Gamma\left[a_{5}\right] \Gamma\left[4-2 \epsilon-a_{13}\right](2 \pi i)^{3}} \int_{-i \infty}^{+i \infty} d \alpha \int_{-i \infty}^{+i \infty} d \beta \int_{-i \infty}^{+i \infty} d \gamma \\
& (-s)^{\left(2-\epsilon-a_{245}-\alpha+\beta-\gamma\right)}(-t)^{\gamma} \Gamma[-\alpha] \Gamma[-\gamma] \Gamma\left[a_{1}+\beta\right] \\
& \frac{\Gamma\left[4-2 \epsilon-a_{113}-\beta\right] \Gamma\left[-2+\epsilon+a_{13}+\beta\right]}{\Gamma\left[5-2 \epsilon-a_{245}+\beta\right]} \\
& \frac{\Gamma\left[-2+\epsilon+a_{245}+\alpha-\beta+\gamma\right]}{\Gamma\left[4-2 \epsilon-a_{22445}-2 \alpha+\beta-2 \gamma\right]} \frac{\Gamma\left[2-\epsilon-a_{245}-\alpha-\gamma\right]}{\Gamma\left[5-2 \epsilon-a_{22445}-2 \alpha+\beta-2 \gamma\right]} \\
& {\left[p_{e} \cdot\left(p_{1}+p_{2}\right) \Gamma\left[5-2 \epsilon-a_{22445}+\beta-2 \gamma\right]\right.} \\
& \Gamma\left[4-2 \epsilon-a_{22445}-2 \alpha+\beta-2 \gamma\right] \\
& \Gamma\left[a_{2}+\gamma\right] \Gamma\left[a_{4}+\gamma\right] \Gamma\left[3-\epsilon-a_{24}-\alpha+\beta-\gamma\right] \\
& +\Gamma\left[4-2 \epsilon-a_{224445}+\beta-2 \gamma\right] \Gamma\left[5-2 \epsilon-a_{22445}-2 \alpha+\beta-2 \gamma\right] \\
& \Gamma\left[2-\epsilon-a_{24}-\alpha+\beta-\gamma\right] \\
& \left.\left(p_{e} \cdot p_{1} \Gamma\left[1+a_{2}+\gamma\right] \Gamma\left[a_{4}+\gamma\right]+p_{e} \cdot p_{3} \Gamma\left[a_{2}+\gamma\right] \Gamma\left[1+a_{4}+\gamma\right]\right)\right]
\end{aligned}
$$

$$
\begin{aligned}
\mathrm{B} 512 \mathrm{~m} 3\left(\mathrm{p}_{\mathrm{e}} \cdot \mathrm{k}_{2}\right)= & \frac{(-1)^{a_{12345}} e^{2 \epsilon \gamma_{E}} \int_{-i \infty}^{+i \infty} d \alpha \int_{-i \infty}^{+i \infty} d \beta \int_{-i \infty}^{+i \infty} d \gamma \int_{-i \infty}^{+i \infty} d \delta}{}+(-s)^{\gamma}(-t)^{\left(4-2 \epsilon-a_{12345}-\beta-\delta-\gamma\right)} \\
& \frac{\Gamma[-\beta] \Gamma[-\gamma] \Gamma[-\delta] \Gamma\left[a_{3}+\alpha+2 \beta\right] \Gamma\left[2-\epsilon-a_{45}+\alpha-\delta-\gamma\right]}{\Gamma\left[7-3 \epsilon-a_{12345}-\beta\right]} \\
& \frac{\Gamma\left[2-\epsilon-a_{13}-\beta\right] \Gamma\left[2-\epsilon-a_{23}-\alpha-\beta\right]}{\Gamma\left[a_{5}-\alpha+2 \gamma\right] \Gamma\left[1+a_{5}-\alpha+2 \gamma\right]} \\
& \Gamma\left[-4+2 \epsilon+a_{12345}+\beta+\delta+\gamma\right] \\
& \left\{\Gamma\left[4-2 \epsilon-a_{1235}-\beta-\delta-\gamma\right]\right. \\
& {\left[\left(p_{e} \cdot p_{2}\right) \Gamma\left[1+a_{5}+\gamma\right] \Gamma[-\alpha+\gamma]-\left(p_{e} \cdot p_{1}\right) \Gamma\left[a_{5}+\gamma\right] \Gamma[1-\alpha+\gamma]\right] } \\
& \Gamma\left[a_{5}-\alpha+2 \gamma\right] \Gamma\left[1+a_{5}-\alpha+2 \delta+2 \gamma\right] \\
& +\left[\left(p_{e} \cdot p_{3}\right)-\left(p_{e} \cdot p_{1}\right)\right] \Gamma\left[5-2 \epsilon-a_{1235}-\beta-\delta-\gamma\right] \\
& \left.\Gamma\left[a_{5}+\gamma\right] \Gamma[-\alpha+\gamma] \Gamma\left[1+a_{5}-\alpha+2 \gamma\right] \Gamma\left[a_{5}-\alpha+2(\delta+\gamma)\right]\right\}
\end{aligned}
$$




$$
\begin{aligned}
& \operatorname{B513m}\left(\mathrm{p}_{\mathrm{e}} \cdot \mathrm{k}_{2}\right)=\frac{(-1)^{a_{12345} e^{2 \epsilon \gamma_{E}}}}{\prod_{j=1}^{5} \Gamma\left[a_{i}\right] \Gamma\left[4-2 \epsilon-a_{123}\right](2 \pi i)^{4}} \int_{-i \infty}^{+i \infty} d \alpha \int_{-i \infty}^{+i \infty} d \beta \int_{-i \infty}^{+i \infty} d \gamma \int_{-i \infty}^{+i \infty} d \delta \\
& (-s)^{4-2 \epsilon-a_{12345}-\alpha-\beta-\delta}(-t)^{\delta} \\
& \Gamma\left[2-\epsilon-a_{13}-\alpha-\gamma\right] \Gamma\left[a_{1}+\gamma\right] \\
& \underline{\Gamma\left[-4+2 \epsilon+a_{12345}+\alpha+\beta+\delta\right]} \\
& \Gamma\left[6-3 \epsilon-a_{12345}-\alpha\right] \\
& \frac{\Gamma[-\alpha] \Gamma[-\beta]}{\Gamma\left[7-3 \epsilon-a_{12345}-\alpha\right]} \Gamma\left[4-2 \epsilon-a_{12345}-\alpha-\gamma-\beta-\delta\right] \\
& \frac{\Gamma[-\delta]}{\Gamma\left[4-2 \epsilon-a_{1123}-2 \alpha-\gamma\right]} \\
& \frac{\Gamma\left[2-\epsilon-a_{12}-\alpha\right]}{\Gamma\left[8-4 \epsilon-a_{112233445}-2 \alpha-\gamma-2 \beta-2 \delta\right]} \\
& \frac{\Gamma\left[4-2 \epsilon-a_{1123}-\gamma\right]}{\Gamma\left[9-4 \epsilon-a_{112233445}-2 \alpha-\gamma-2 \beta-2 \delta\right]} \\
& \left(-\left(\left(p_{e} \cdot p_{1}\right) \Gamma\left[7-3 \epsilon-a_{12345}-\alpha\right] \Gamma\left[8-4 \epsilon-a_{112233445}-2 \alpha-\gamma-2 \delta\right]\right.\right. \\
& \Gamma\left[9-4 \epsilon-a_{112233445}-2 \alpha-\gamma-2 \beta-2 \delta\right] \Gamma\left[4-2 \epsilon-a_{1234}-\alpha-\beta-\delta\right] \\
& \left.\Gamma\left[a_{4}+\delta\right] \Gamma\left[-2+\epsilon+a_{123}+\alpha+\gamma+\delta\right]\right) \\
& +\Gamma\left[6-3 \epsilon-a_{12345}-\alpha\right] \\
& \left(\left(p_{e} \cdot\left(p_{1}+p_{2}\right)\right) \Gamma\left[9-4 \epsilon-a_{112233445}-2 \alpha-\gamma-2 \delta\right]\right. \\
& \Gamma\left[8-4 \epsilon-a_{112233445}-2 \alpha-\gamma-2 \beta-2 \delta\right] \\
& \Gamma\left[5-2 \epsilon-a_{1234}-\alpha-\beta-\delta\right] \Gamma\left[a_{4}+\delta\right] \\
& \Gamma\left[-2+\epsilon+a_{123}+\alpha+\gamma+\delta\right] \\
& +\Gamma\left[8-4 \epsilon-a_{112233445}-2 \alpha-\gamma-2 \delta\right] \\
& \Gamma\left[9-4 \epsilon-a_{112233445}-2 \alpha-\gamma-2 \beta-2 * \delta\right] \\
& \Gamma\left[4-2 \epsilon-a_{1234}-\alpha-\beta-\delta\right] \\
& \left(\left(p_{e} \cdot p_{3}\right) \Gamma\left[1+a_{4}+\delta\right] \Gamma\left[-2+\epsilon+a_{123}+\alpha+\gamma+\delta\right]\right. \\
& \left.\left.\left.+\left(p_{e} \cdot p_{1}\right) \Gamma\left[a_{4}+\delta\right] \Gamma\left[-1+\epsilon+a_{123}+\alpha+\gamma+\delta\right]\right)\right)\right) \\
& \operatorname{B513m}\left(\mathrm{p}_{\mathrm{e}} \cdot \mathrm{k}_{1}\right)=\frac{(-1)^{a_{12345}} e^{2 \epsilon \gamma_{E}}}{\prod_{j=1}^{5} \Gamma\left[a_{i}\right] \Gamma\left[5-2 \epsilon-a_{123}\right](2 \pi i)^{4}} \int_{-i \infty}^{+i \infty} d \alpha \int_{-i \infty}^{+i \infty} d \beta \int_{-i \infty}^{+i \infty} d \gamma \int_{-i \infty}^{+i \infty} d \delta \\
& (-s)^{(4-2 \epsilon)-a_{12345}-\alpha-\beta-\delta}(-t)^{\delta} \\
& \frac{\Gamma\left[-4+2 \epsilon+a_{12345}+\alpha+\beta+\delta\right]}{\Gamma\left[6-3 \epsilon-a_{12345}-\alpha\right]} \\
& \frac{\Gamma[-\alpha] \Gamma[-\beta]}{\Gamma\left[7-3 \epsilon-a_{12345}-\alpha\right] \Gamma\left[5-2 \epsilon-a_{123}\right]} \\
& \frac{\Gamma[-\delta]}{\Gamma\left[4-2 \epsilon-a_{1123}-2 \alpha-\gamma\right] \Gamma\left[5-2 \epsilon-a_{1123}-2 \alpha-\gamma\right]}
\end{aligned}
$$




$$
\begin{aligned}
& \frac{\Gamma\left[2-\epsilon-a_{13}-\alpha-\gamma\right]}{\Gamma\left[8-4 \epsilon-a_{112233445}-2 \alpha-2 \beta-2 \delta-\gamma\right]} \\
& \frac{\Gamma\left[4-2 \epsilon-a_{12345}-\alpha-\beta-\delta-\gamma\right]}{\Gamma\left[9-4 \epsilon-a_{112233445}-2 \alpha-2 \beta-2 \delta-\gamma\right]} \\
& \left\{\left(p_{e} \cdot p_{3}\right) \Gamma\left[1+a_{4}+\delta\right] \Gamma\left[6-3 \epsilon-a_{12345}-\alpha\right]\right. \\
& \Gamma\left[4-2 \epsilon-a_{1234}-\alpha-\beta-\delta\right] \Gamma\left[3-\epsilon-a_{12}-\alpha\right] \\
& \Gamma\left[8-4 \epsilon-a_{112233445}-2 \alpha-2 \delta-\gamma\right] \\
& \Gamma\left[9-4 \epsilon-a_{112233445}-2 \alpha-2 \beta-2 \delta-\gamma\right] \\
& \Gamma\left[5-2 \epsilon-a_{1123}-\gamma\right] \Gamma\left[4-2 \epsilon-a_{1123}-2 \alpha-\gamma\right] \\
& \Gamma\left[a_{1}+\gamma\right] \Gamma\left[-2+\epsilon+a_{123}+\alpha+\delta+\gamma\right]+\Gamma\left[a_{4}+\delta\right] \\
& {\left[-\left(p_{e} \cdot p_{1}\right) \Gamma\left[7-3 \epsilon-a_{12345}-\alpha\right]\right.} \\
& \Gamma\left[4-2 \epsilon-a_{1234}-\alpha-\beta-\delta\right] \\
& \Gamma\left[8-4 \epsilon-a_{112233445}-2 \alpha-2 \delta-\gamma\right] \\
& \Gamma\left[9-4 \epsilon-a_{112233445}-2 \alpha-2 \beta-2 \delta-\gamma\right] \\
& {\left[\Gamma\left[3-\epsilon-a_{12}-\alpha\right] \Gamma\left[5-2 \epsilon-a_{1123}-\gamma\right]\right.} \\
& \Gamma\left[4-2 \epsilon-a_{1123}-2 \alpha-\gamma\right] \Gamma\left[a_{1}+\gamma\right] \\
& +\Gamma\left[2-\epsilon-a_{12}-\alpha\right] \Gamma\left[4-2 \epsilon-a_{1123}-\gamma\right] \\
& \left.\Gamma\left[5-2 \epsilon-a_{1123}-2 \alpha-\gamma\right] \Gamma\left[1+a_{1}+\gamma\right]\right] \\
& \Gamma\left[-2+\epsilon+a_{123}+\alpha+\delta+\gamma\right] \\
& +\Gamma\left[6-3 \epsilon-a_{12345}-\alpha\right] \Gamma\left[3-\epsilon-a_{12}-\alpha\right] \\
& \Gamma\left[5-2 \epsilon-a_{1123}-\gamma\right] \Gamma\left[4-2 \epsilon-a_{1123}-2 \alpha-\gamma\right] \\
& \Gamma\left[a_{1}+\gamma\right]\left[\left(\left(p_{e} \cdot\left(p_{1}+p_{2}\right)\right) \Gamma\left[5-2 \epsilon-a_{1234}-\alpha-\beta-\delta\right]\right.\right. \\
& \Gamma\left[9-4 \epsilon-a_{112233445}-2 \alpha-2 \delta-\gamma\right] \\
& \Gamma\left[8-4 \epsilon-a_{112233445}-2 \alpha-2 \beta-2 \delta-\gamma\right] \\
& \Gamma\left[-2+\epsilon+a_{123}+\alpha+\delta+\gamma\right] \\
& +\left(p_{e} \cdot p_{1}\right) \Gamma\left[4-2 \epsilon-a_{1234}-\alpha-\beta-\delta\right] \\
& \Gamma\left[8-4 \epsilon-a_{112233445}-2 \alpha-2 \delta-\gamma\right] \\
& \Gamma\left[9-4 \epsilon-a_{112233445}-2 \alpha-2 \beta-2 \delta-\gamma\right] \\
& \left.\left.\Gamma\left[-1+\epsilon+a_{123}+\alpha+\delta+\gamma\right]\right]\right\}
\end{aligned}
$$

\section{References}

[1] A. Penin, Phys. Rev. Lett. 95 (2005) 010408;

A. Penin, Nucl. Phys. B 734 (2006) 185. 
[2] A. Arbuzov, V. Fadin, E. Kuraev, L. Lipatov, N. Merenkov and L. Trentadue, Nucl. Phys. Proc. Suppl. 51C (1996) 154;

S. Jadach, M. Melles, B. Ward and S. Yost, Nucl. Phys. Proc. Suppl. 51C (1996) 164;

S. Jadach, "Theoretical error of luminosity cross section at LEP", hep-ph/0306083,

A. Arbuzov and E. Scherbakova, "Next-to-leading order corrections to Bhabha scattering in renormalization group approach. I: Soft and virtual photonic contributions", hep-ph/0602119.

[3] Z. Bern, L. Dixon and A. Ghinculov, Phys. Rev. D 63 (2001) 053007.

[4] R. Bonciani, A. Ferroglia, P. Mastrolia, E. Remiddi and J. van der Bij, Nucl. Phys. B681 (2004) 261.

[5] M. Czakon, J. Gluza and T. Riemann, Phys. Rev. D 71 (2005) 073009;

[6] M. Czakon, J. Gluza and T. Riemann, Nucl. Phys. Proc. Suppl. 135 (2004) 83.

[7] M. Czakon, J. Gluza and T. Riemann, http://www-zeuthen.desy.de/theory/research/bhabha/.

[8] V. Smirnov, Phys. Lett. B 524 (2002) 129.

[9] G. Heinrich and V. Smirnov, Phys. Lett. B 598 (2004) 55.

[10] M. Czakon, "Automatized analytic continuation of Mellin-Barnes integrals", to appear in Comput. Phys. Commun., hep-ph/0511200.

[11] V. Smirnov, Phys. Lett. B 460 (1999) 397.

[12] S. Moch and P. Uwer, "XSummer: Transcendental functions and symbolic summation in Form", to appear in Comput. Phys. Commun., arXiv math-ph/0508008.

[13] H. Ferguson and D. Bailey, RNR Technical Report RNR-91-032, NASA Ames Research Center (1991);

D. Bailey, J. Borwein and R. Girgensohn, Experimental Mathematics 3 (1994) 17;

H. Ferguson, D. Bailey and S. Arno, Mathematics of Computation 68 (1990) 351.

[14] S. Actis, M. Czakon, J. Gluza, T. Riemann, in preparation. 